\title{
Association Between miR-30a/SNAl1 Gene Polymorphisms and the Risk of Diabetic Kidney Disease
}

\section{CaiLian Yang}

First Affiliated Hospital of Chongqing Medical University

\section{Tao Tong}

First Affiliated Hospital of Chongqing Medical University

\section{MingFang Sun}

First Affiliated Hospital of Chongqing Medical University

Bo Zhou ( $D$ zhoubo915@126.com)

First Affiliated Hospital of Chongqing Medical University

\section{Research Article}

Keywords: diabetic kidney disease, microRNAs, single nucleotide polymorphism, miR-30a, SNAI1

Posted Date: December 23rd, 2020

DOI: https://doi.org/10.21203/rs.3.rs-127615/v1

License: (9) This work is licensed under a Creative Commons Attribution 4.0 International License. Read Full License 


\section{Association between miR-30a/SNAI1 gene polymorphisms and the risk of diabetic kidney disease}

Cailian Yang ${ }^{1,2}$, Tao Tong ${ }^{1,3}$, Mingfang Sun ${ }^{1,4}$, Bo Zhou ${ }^{1,5}$.

1.Department of Endocrinology, the First Affiliated Hospital of Chongqing Medical University,Chongqing,400000,China. 2.1808622838@qq.com; 3.tong tong123@163.com; 4.189659280@qq.com; 5.zhoubo915@126.com

\section{Abstract}

Background:Diabetic kidney disease is a leading cause of end-stage kidney disease worldwide, its incidence is still increasing and the precise mechanism is not yet fully understood.This study aimed to investigate the possible association of miR-30a and its potential target gene, SNAI1, polymorphisms with diabetic kidney disease in patients with type 2 diabetes mellitus.

Methods:This case-control is study included 240 type 2 diabetes mellitus patients with diabetic kidney disease and 280 patients without diabetic kidney disease.Genotyping of miR-30a and SNAl1 polymorphisms were performed by allelic discrimination assay with Taq-Man-MGB probes.

Result:The results demonstrated that the CC genotype of rs2222722 in miR-30a was associated with an increased risk of diabetic kidney disease in Chinese type 2 diabetes mellitus patients $(\mathrm{OR}=2.81,95 \% \mathrm{Cl} 1.58-4.97, \mathrm{p}<0.001$ ). Stratified analyses revealed that the miR-30a CC genotype was strongly associated with an increased risk of diabetic kidney disease in subjects who were young ( $<65$ years), male, and had a low body mass index, as well as those with poor glycemic control. What's more, CC genotype carriers were more likely to exhibit decreased estimated glomerular filtration rate levels and urinary protein production. In addition, the GG genotype of SNAII was also associated with the development of diabetic kidney disease, and the risk was 1.73 times higher than that in $A A+A G$ genotype carriers $(95 \% \mathrm{Cl} 1.01-2.96, p=0.046)$. Multiple logistic regression analysis showed that the miR-30a CC and SNAI1 GG genotypes were indispensable and dangerous contributing factors to the development of diabetic kidney disease.

Conclusion: The CC genotype of miR-30a rs2222722 and GG genotype of SNAI1 rs1543442 might be associated with diabetic kidney disease risk in Chinese type 2 diabetes mellitus patients.

Keywords: diabetic kidney disease, microRNAs, single nucleotide polymorphism, miR-30a, SNAI1

\section{Introduction}

Diabetic kidney disease (DKD), characterized by albuminuria or reduced glomerular filtration rate (GFR), is a leading cause of end-stage kidney disease (ESKD) worldwide[1,2]. Despite the current variety of antihyperglycemic and antihypertensive treatments and new intervention strategies, the incidence of DKD is still increasing[3]. Previous studies have suggested that glucose, lipid metabolism disorders, oxidative stress, inflammatory reactions and multiple cytokines are closely related to the development of DKD, but the precise mechanism is not yet fully understood[4,5]. Both clinical and epidemiological studies have demonstrated familial aggregation of DKD in different ethnic groups, indicating that genetic and epigenetic factors play an important role in the development of DKD[6-10]. Therefore, it is important to identify potential susceptibility genes and gene loci while exploring the molecular genetics of DKD to facilitate early diagnosis and identify potential targets for its treatment to prevent ESKD. 
To date, many studies have indicated that microRNAs (miRNAs) can affect various gene expression levels in kidney diseases[11-14]. MiRNAs are a large family of endogenous, singlestranded, small noncoding RNAs of 18-25 nucleotides in length that downregulate target gene expression by binding to the $3^{\prime}$-untranslated region ( $3^{\prime} U T R$ ) of target mRNAs, leading to mRNA degradation or translational inhibition[15]. They broadly participate in diverse physiological processes, including cell proliferation, cell apoptosis, cell differentiation, immunity, and metabolism[16]. Among them, miR-30a expression is enriched in the kidney and is receiving increasing attention[17,18]. Peng, R. et al. found that miR-30a was significantly downregulated in cultured podocytes treated with Adriamycin (ADR) in vivo and in vitro. In addition, miR-30a inhibits the epithelial to mesenchymal transition (EMT) to protect podocyte functions by targeting NFATc3[19]. Furthermore, Zhao et al. found that upregulating miR-30a protects podocytes from angiotensin II (AngII)-induced cytoskeletal damage and apoptosis in vitro[20]. More importantly, a recent study revealed that miR-30a was downregulated in glomeruli and proximal tubules from human kidney needle biopsy specimens in different types of kidney diseases, including DKD, focal segmental glomerulosclerosis, and membrane proliferative glomerulonephritis[21]. Furthermore, our previous research demonstrated that SNAI1, a potential target of miR-30a (http://www.targetscan.org/, https://www.mirbase.org/), was significantly upregulated in the podocyte EMT process induced by high glucose in vitro and did not return to normal levels, even after normal glucose culture[22]. Recently, Zheng et al. confirmed that miR-30a inhibits the EMT via reduction of SNAI1, leading to the suppression of hepatic stellate cell activation in liver fibrosis[23]. Moreover, upregulation of miR-30a represses protein expression of SNAI1, alphasmooth muscle actin (SMA) and vimentin and downregulates the EMT induced by high glucose and TGF-beta2 in a diabetic cataract model[24]. Above all. miR-30a/SNAI1 may play a crucial role in the pathogenesis of DKD.

Polymorphisms of miR-30a and SNAI1 are significantly associated with the development of primary nephrotic syndrome[25] and various tumorigenesis[26-30]. However, to our knowledge, the correlation of miR-30a and SNAI1 variants with the risk of DKD has not been examined. In this study, we aimed to investigate the association of these genetic polymorphisms with DKD in Chinese type 2 diabetes mellitus (T2DM) patients by conducting a case-control study.

\section{Materials and Methods}

\section{Subjects}

In this study, patients with T2DM were recruited from the First Affiliated Hospital of ChongQing Medical University. Among them, 280 patients with T2DM without DKD were defined as the control group. The remaining 240 patients with DKD were defined as the case group. The diagnosis of DKD was established according to the 2012 Kidney Disease Improving Global Outcomes (KDIGO) guidelines. The exclusion criteria were as follows: history of an acute stress state, such as surgery, hyperglycemic crisis, heart failure, or urinary tract infection; history of various tumors; and history of kidney damage caused by other reasons. 
We collected the following clinical and laboratory variables for all patients: age, sex, systolic blood pressure (SBP), diastolic blood pressure (DBP), pulse pressure (PP), duration of T2DM and hypertension, body mass index (BMI), smoking and drinking status,fasting plasma glucose (FPG), glycosylated hemoglobin (HbA1c), total cholesterol (TC), triglycerides (TGs), high-density lipoprotein cholesterol (HDL-C), low-density lipoprotein cholesterol (LDL-C), C-reactive protein (CRP), fibrinogen (FIB), estimated GFR (eGFR), urinary albumin/creatinine ratio (ACR) and medications. All biochemical analyses were performed in the hospital laboratory by standard procedures.

\section{Single Nucleotide Polymorphism Selection and Genotyping}

Genomic DNA was extracted from $400 \mu$ l of peripheral blood using the QIAamp DNA Blood Mini Kit (Qiagen, Hilden, Germany) in accordance with the supplier's protocol. The DNA concentration was measured by a NanoDrop 2000C spectrophotometer (Thermo Scientific, Waltham, MA, USA).

Polymerase chain reaction (PCR) amplification was performed in a $25 \mu$ reaction mixture containing $2 \mu \mathrm{l}$ of DNA, $12.5 \mu \mathrm{l}$ of TaqMan PCR enzyme, $10 \mu \mathrm{l}$ of deionized water, $1.0 \mu \mathrm{l}$ of TaqMan FAM/HEX probes, and $1 \mu \mathrm{l}$ of upstream and downstream primers. The amplification conditions were 40 cycles of $95^{\circ} \mathrm{C}$ for $30 \mathrm{~s}, 95^{\circ} \mathrm{C}$ for $5 \mathrm{~s}$, and at $60^{\circ} \mathrm{C}$ for $30 \mathrm{~s}$. The primers and probes used to detect single nucleotide polymorphisms (SNPs) were provided by Takara Bio. The product was sequenced using a CFX96 Real-Time PCR Detection system (BIO-RAD). A random selection of amplified samples was sent to Sangon Biotech(shang hai) for sequencing to verify the accuracy of the genotype determined by the TaqMan method. The results showed that the accuracy of the TaqMan detection method in this study was as high as $100 \%$.

\section{Statistical Analysis}

Statistical analysis was performed with SPSS 21.0 software. Continuous variables are expressed as the mean \pm standard deviation (for normally distributed data) or as the median (for data with a skewed distribution). Hardy-Weinberg equilibrium analysis of both SNPs was conducted using the Chi-squared test. Differences between groups were compared using an unpaired Student's t-test or analysis of variance (ANOVA) for normally distributed continuous variables and the Pearson $\chi^{2}$ test for categorical variables. Associations of miR-30a and SNAl1 polymorphisms with atherosclerosis risk were assessed by unconditional logistic regression analysis and adjusted for possible confounders identified by the univariate analysis. Interactions between the genotypes and clinical parameters were evaluated using stepwise logistic regression analysis for categorical variables (ACR and eGFR). A p-value of $<0.05$ was considered statistically significant, and DKD susceptibility was estimated with odds ratios (ORs) and $95 \%$ confidence intervals (Cls) based on logistic regression analysis. 


\section{Results}

\section{Comparison of General Characteristics of the Subjects}

The clinical and biochemical parameters of the T2DM and DKD groups are shown in Table 1. Compared to the T2DM group, patients with DKD were older and had higher SBP and PP, longer duration of diabetes and hypertension and lower smoking and drinking rates $(p<0.05)$. In addition, biochemical parameter analysis showed that patients in the DKD group had significantly higher FIB, CRP, and ACR values and lower eGFR levels, and patients with DKD were more likely to receive angiotensin-converting enzyme inhibitor (ACEI)/angiotensin receptor blocker (ARB), antiplatelet and lipid-lowering therapies. However, there were no significant differences in sex, BMI, DBP, fasting plasma glucose (FPG), HbA1C, TC, TG, HDL-C, or LDL-C between T2DM and DKD patients.

\section{Genotype Distributions}

The miR-30a rs2222722 (C>T) and SNAI1 rs1543442 (A>G) genotypes were both consistent with Hardy-Weinberg equilibrium ( $p>0.05$ ). The frequencies of the $C C$ genotype and $C$ allele of miR30a rs2222722 (C>T) in the DKD group were significantly higher than those in the T2DM group (genotype, $p=0$ 012; allele, $p=0$ 007). Meanwhile, in the DKD group, the proportions of the GG genotype and $\mathrm{G}$ allele of SNAI1 rs1543442 ( $>\mathrm{G}$ ) were higher than those in the T2DM group, but there was no significant difference between the two groups $(p>0.05)$ (Table 2$)$.

Dominant and recessive models were created to analyze the relationship between the miR-30a rs2222722 (C>T) and SNAI1 rs1543442 (A>G) polymorphisms and the DKD risk. In the miR-30a rs2222722 (C>T) recessive model, the risk of DKD in CC genotype carriers was increased 1.81-fold (adjusted 95\% $\mathrm{Cl}$ 1.58-4.97, $\mathrm{p}<0.001$ ) compared to the $\mathrm{CT}+\mathrm{TT}$ genotype, even after adjusting for age, sex, BMI, duration of diabetes, hypertension, SBP, DBP, PP, FPG, HbA1C, TC, TG, HDL-C, LDL-C, $\mathrm{FIB}, \mathrm{CRP}$, and $\mathrm{ACEI} / \mathrm{ARB}$, antiplatelet and lipid-lowering drug use. However, there was no significant change in the risk for DKD in the dominant model $(p>0.05)$. Meanwhile, in the recessive model of SNAI1 rs1543442 $(A>G)$, the risk of DKD was significantly increased by the GG genotype, even after the relevant confounding factors were adjusted (adjusted $\mathrm{OR}=1.73,95 \% \mathrm{Cl}$

1.01-2.96, $p=0.046$ ) (Table 3).

\section{Stratified Analysis of Clinical Features}

Patients in the DKD and T2DM groups were stratified according to age, sex, duration of diabetes, HbA1C and BMI levels, and the correlation between the miR-30a rs2222722 (C>T) and SNAI1 rs1543442 (A>G) genotypes and DKD risk in different populations was analyzed (Table 4). The results showed that in miR-30a rs2222722 (C>T) CC genotype carriers, the risk of DKD was significantly increased $(p<0.05)$ if patients were young $(<65$ years), had worse glycemic control $(\mathrm{HbA} 1 \mathrm{c}>8 \%)$ and had a low BMI $\left(<24 \mathrm{~kg} / \mathrm{m}^{2}\right)$. Furthermore, in the $\mathrm{GG}$ genotype of SNAI1 rs1543442 (A>G) carriers, the DKD risk was significantly higher in males and those with a diabetes duration $<10$ years, $\mathrm{HbA} 1 \mathrm{c}<8 \%$ and $\mathrm{BMI}<24 \mathrm{~kg} / \mathrm{m}^{2}$. 


\section{Relationship between the miR-30a rs2222722 (C>T) and SNAI1 rs1543442 (A>G) Genotypes and the Risk of Urinary Albumin and Decreased eGFR}

The present results indicate that the miR-30a and SNAI1 polymorphisms are closely related to development of DKD. According to the KDIGO guidelines, urinary albumin and eGFR are the main diagnostic basis for DKD. Because their mechanisms may be different, we further analyzed the relationships of different phenotypes with urinary albumin and eGFR. After adjusting for age and sex, a relationship was observed in miR-30a rs2222722 (C>T) recessive model ( $T T+C T$ vs CC), in which CC genotype carriers were associated with an increased incidence of urinary albumin and decreased eGFR compared to TT+CT genotype carriers (ACR: OR $=1.54 .95 \% \mathrm{Cl} 1.05-2.26, p=$ 0.026; eGFR: $O R=1.62,95 \% \mathrm{Cl} 1.11-2.37, \mathrm{p}=0.012$ ). Moreover, in $\mathrm{C}$ allele carriers, the risk of urinary albumin and decreased eGFR was higher than that in T allele carriers (ACR: OR $=1.4195 \%$ $\mathrm{Cl} 1.06-1.88, \mathrm{p}=0.019$; eGFR: $\mathrm{OR}=1.38,95 \% \mathrm{Cl} 1.04-1.83, \mathrm{p}=0.027)$. However, there was no significant relationship between the risk of urinary albumin and eGFR decrease and the GG genotype or G allele carriers in the SNAI1 rs1543442 (A>G) recessive and dominant models (Table $5)$.

\section{Nonconditional Logistic Regression Analysis}

Nonconditional logistic regression analysis was performed with or without DKD as the dependent variable and age, sex, duration of diabetes, duration of hypertension, BMI, SBP, DBP, PP, smoking, alcohol use, FPG, HbA1c, TC, TG, HDL-C, LDL-C, FIB, CRP, ACEI/ARB use, lipid-lowering drug use, antiplatelet use, and miR-30a and SNAI1 genotypes as the independent variables. The results showed that the CC genotype of miR-30a rs2222722 and the GG genotype of SNAl1 rs1543442 were independent risk factors for DKD (Table 6).

\section{Synergistic Effect of MiR-30a rs2222722 (C>T) and SNAl1 rs15434442 (A>G) Polymorphisms on DKD}

We further analyzed the synergistic effect of both SNPs on DKD (Table 7). When the miR-30a rs2222722 CT/TT + SNAI1 rs1543442 AA genotype was used as the reference, patients with the miR-30a rs2222722 CT/TT + SNAI1 rs1543442 AG genotype exhibited a lower risk of DKD (OR: 0.46; $95 \% \mathrm{Cl}$ 0.27-0.76; $p=0.003$ ). However, no difference was observed in other genotype combinations of miR-30a rs2222722 (C>T) and SNAI1 rs1543442 (A>G).

\section{Discussion}

According to the International Diabetes Federation (IDF) data, in $2015,7.2-11.4 \%$ of people worldwide suffered from diabetes, and 44.4\% of T2DM patients will progress to DKD over the next three decades[31]. Furthermore, DKD is associated with a significant increase in cardiovascular and all-cause mortality. Most of the cardiovascular and all-cause mortality caused by diabetes occurs in patients with kidney disease[32]. Although current clinical treatments are promising for DKD, it is still difficult to halt the progression of DKD in T2DM patients, primarily due to genetic 
and epigenetic variabilities[33-35]. Recently, increasing studies have also shown that genetic factors play an important role in the development of DKD[36-37].

In our study, miR-30a rs2222722 (C>T) locus SNPs were significantly associated with the risk of DKD. Patients with the CC genotype had a 2.81 -fold higher risk of DKD than patients with CT and TT genotypes, even after adjusting for possible confounding factors. We further explored the relationship between the miR-30a rs2222722 (C>T) polymorphism and urinary albumin and eGFR levels, and the results demonstrated that $\mathrm{CC}$ genotype carriers are more likely to have decreased eGFR levels and urinary protein production.

The rs2222722 (C>T) polymorphism is located in the promoter region of the miR-30a gene, and the database predicted that it may be a transcription factor binding site, affecting the transcription level of miR-30a[38]. MiR-30a participates in different biochemical processes in various ways, such as the EMT process[16, 34]. The EMT is often thought to be the result of epithelial cells undergoing a crucial phenotypic conversion. During this transition, epithelial cells attenuate their intercellular junctions and adhesions, lose cell polarity and primordial morphology, and strengthen their cell motility and migratory capabilities[39-41]. Lee and colleagues reported that miR-30a plays a role in the development of colorectal cancer by modulating the EMT and cell motility by directly targeting TM4SF1[42]. Similarly, Zhou and coworkers suggested that miR-30a inhibits the EMT via a reduction in Snai1, leading to the suppression of hepatic stellate cell activation in liver fibrosis[43]. Furthermore, the EMT might be a primary cause of podocyte dysfunction, proteinuria, and glomerulosclerosis[44]. In ADR-treated podocytes, alterations in epithelial markers were upregulated in the presence of miR-30a mimics and were reduced when cells were transfected with a miR-30a inhibitor. In contrast, mesenchymal markers of the EMT in podocytes were suppressed in the presence of miR-30a mimics and enhanced when cells were transfected with a miR-30a inhibitor, demonstrating that miR-30a might represent a potential target for preventing the EMT in podocytes[19]. In addition, in an Angll-induced podocyte injury model, miR-30a was downregulated in mouse podocytes, and when exogenous miR-30a was delivered to the podocytes via lentivirus injection, glomerular damage and podocyte injury were significantly ameliorated. This indicates that the lentiviral miR-30a delivered into podocytes may have exerted a direct protective effect[20].

In addition, the GG genotype of SNAI1 rs1543442 was associated with DKD, and the risk of DKD was 1.73 times higher than that of AA+AG genotypes in the recessive model. rs1543442 is located in the $3^{\prime} U T R$ region of the SNAI1 gene, which is the site of microRNA regulation of gene expression. As a potential target of miR-30a, the SNAl1 zinc finger domain combines with the CAGGTG sequence within the E-cadherin promoter region, downregulating E-cadherin expression and initiating the EMT process[45]. As reported, SNAI1 activation repressed the tubular epithelial phenotype through downregulation of E-cadherin and induced renal fibrosis with concomitant expression of vimentin, alpha-SMA, and collagen I. In addition, inhibiting SNAI1 activity provides a specific method to prevent or reverse renal fibrosis[46]. However, we did not find an association 
between SNAI1 rs1543442 polymorphisms and urinary albumin or eGFR levels in our population; therefore, the impact of SNAI1 polymorphisms in DKD should be evaluated further.

We also performed stratified analyses of DKD risk according to age, sex, diabetes duration, $\mathrm{BMI}$ and $\mathrm{HbA} 1 \mathrm{C}$ level. The results revealed that the risk for DKD in patients with the miR-30a CC genotype was more pronounced in patients who were young ( $<65$ years), were male, had a low BMI $\left(<24 \mathrm{~kg} / \mathrm{m}^{2}\right)$ and had poor glycemic control (HbA1c\%). In SNAl1 rs1543442 (A>G) GG genotype carriers, DKD risk was significantly higher in patients who were male and had a duration of diabetes $<10$ years, $\mathrm{HbA} 1 \mathrm{c}<8 \%$ and $\mathrm{BMI}<24 \mathrm{~kg} / \mathrm{m}^{2}$. This indicates that the correlation of miR30a and SNAI1 SNPs with DKD risk is affected by clinical features. The results of this study may be clinically useful in different populations. Moreover, multiple logistic regression analysis revealed that the CC (miR-30a rs2222722) and GG (SNAI1 rs1543442) genotypes were indispensable and dangerous contributing factors for the development of DKD. The gene-gene interactions between miR-30a rs2222722 and SNAI1 rs1543442 polymorphisms were further analyzed as well, but no combined effects of these two genes on enhancing the risk of DKD were detected. Further research is needed to confirm these findings.

In summary, our study suggests that miR-30a rs2222722 (C>T) and SNAl1 rs1543442 (A>G) locus SNPs are associated with the risk of DKD in patients with type 2 diabetes in the Chinese Han population. MiR-30a and SNAI1 might represent potential promising biomarkers of DKD development and progression. Nevertheless, there are some limitations to this study that should be considered. First, the sample size in this study is small, and more people with type 2 diabetes should be recruited to form a larger study cohort, which will improve the statistical power. Second, although the two SNPs in miR-30a and SNAI1 were identified as susceptibility variants to DKD in patients with type 2 diabetes in a Chinese Han population, it is important to screen more sites to clarify the interaction between SNPs and DKD. Third, since this is the first study to explore the association of miR-30a and SNAI1 gene polymorphisms with DKD, our results need to be replicated in other independent cohorts in the future.

\section{Ethics approval and consent to participate}

This study was approved by the national medical ethics committee and performed in compliance with the Declaration of Helsinki. All participants were provided informed consent.

\section{Consent for publication}

Not applicable

\section{Conflicts of Interest}

We declare that there are no conflicts of interest.

\section{Data Availability}

The data used to support the findings of this study are available from the corresponding authors upon reasonable request.

\section{Acknowledgments}

This study was supported by the First Affiliated Hospital of ChongQing Medical University and the ChongQing Key Laboratory of Translational Medicine in Major Metabolic Diseases. 


\section{Funding}

Funding was provided by the National Natural Science Foundation of China (81370940).

\section{Authors' contributions}

Cailian Yang and Bo Zhou participated in the study design, analysis, writing and editing of the manuscript, MingFang Sun and Tao Tong participated in data generation and study analysis.

\section{References}

1. Alicic RZ, Rooney MT, Tuttle KR: Diabetic kidney disease: Challenges, progress, and possibilities. Clin J Am Soc Nephrol, 2017; 12(12): 2032-45

2. Tuttle, K., Bakris, G., Bilous, R., Chiang, J., de Boer, I., Goldstein-Fuchs, J., . . Molitch, M. (2014). Diabetic kidney disease: a report from an ADA Consensus Conference. American journal of kidney diseases : the official journal of the National Kidney Foundation, 64(4), 510-533.

3. Sustained effect of intensive treatment of type 1 diabetes mellitus on development and progression of diabetic nephropathy: the Epidemiology of Diabetes Interventions and Complications (EDIC) study.[J] JAMA. 2003. 290(16). 2159-2167

4. Navarro-Gonzalez JF, Mora-Fernandez C, Muros de Fuentes M, Garcia-Perez J. 2011 Inflammatory molecules and pathways in the pathogenesis of diabetic nephropathy. Nat. Rev.

Nephrol.7, 327-340.

5. Gheith O, Farouk N, Nampoory N, Halim MA, Al-Otaibi T. 2016 Diabetic kidney disease: worldwide difference of prevalence and risk factors. J. Nephropharmacol.5, 49-56.

6. Sun YM, Su Y, Li J et al. Recent advances in understanding the biochemical and molecular mechanism of diabetic nephropathy. Biochem Biophys Res Commun 2013; 433: 359-361

7. Brorsson C, Pociot F: Genetics of diabetic nephropathy in diverse ethnic groups. Contrib Nephrol, 2011; 170: 8-18

8. Wei L, Xiao Y, Li L et al: The Susceptibility genes in diabetic nephropathy. Kidney Dis (Basel), 2018; 4(4): 226-37

9. Gu HF: Genetic and epigenetic studies in diabetic kidney disease. Front Genet, 2019; 10: 507 10. Agius E, Attard G, Shakespeare L et al: Familial factors in diabetic nephropathy: An offspring study. Diabet Med, 2006; 23: 331-34

11. Chen, Y.Q.; Wang, X.X.; Yao, X.M.; Zhang, D.L.; Yang, X.F.; Tian, S.F.; Wang, N.S. Microrna-195 promotes apoptosis in mouse podocytes via enhanced caspase activity driven by BCL2 insufficiency. Am. J. Nephrol. 2011, 34, 549-559.

12. Long, J.; Wang, Y.; Wang, W.; Chang, B.H.; Danesh, F.R. Identification of microrna-93 as a novel regulator of vascular endothelial growth factor in hyperglycemic conditions. J. Biol. Chem. 2010, 285, 23457-23465.

13. Du, R.; Sun, W.; Xia, L.; Zhao, A.; Yu, Y.; Zhao, L.; Wang, H.; Huang, C.; Sun, S. Hypoxia-induced down-regulation of microrna-34a promotes EMT by targeting the notch signaling pathway in tubular epithelial cells. PLoS ONE 2012, 7, e30771.

14. Tang, O.; Chen, X.M.; Shen, S.; Hahn, M.; Pollock, C.A. Mirna-200b represses transforming growth factor- $\beta 1$-induced EMT and fibronectin expression in kidney proximal tubular cells. Am. J. Physiol. Ren. Physiol. 2013, 304, F1266-F1273. 
15. Bong, I. P. N., Ng, C. C., Baharuddin, P., and Zakaria, Z. (2017). MicroRNA expression patterns and target prediction in multiple myeloma development and malignancy. Genes Genom, 39(5), 533-540.

16. Ferland-McCollough, D., Ozanne, S.E., Siddle, K., Willis, A.E., and Bushell, M. (2010). The involvement of microRNAs in type 2 diabetes. Biochem Soc Trans 38, 1565-1570.

17. Sun, Y., Koo, S., White, N., Peralta, E., Esau, C., Dean, N.M., et al. (2004). Development of a micro-array to detect human and mouse microRNAs and characterization of expression in human organs. Nucleic acids Res 32, e188.

18. Agrawal, R., Tran, U., and Wessely O. (2009). The miR-30 miRNA family regulates Xenopus pronephros development and targets the transcription factor Xlim1/Lhx1. Development 136, 3927-3936.

19. Peng, R., Zhou, L., Zhou, Y., Zhao, Y., Li, Q., Ni, D., ... Zhou, Q. (2015). MiR-30a Inhibits the Epithelial-Mesenchymal Transition of Podocytes through Downregulation of NFATc3.

International Journal of Molecular Sciences, 16(10), 24032-24047.

20. Zhao yue,Wu junnan,Zhang mingchao,Angiotensin II induces calcium/calcineurin signaling and podocyte injury by downregulating microRNA-30 family members.[J]. Journal of molecular medicine (Berlin, Germany), 2017, 95(8): 887-898.

21. Baker, M. A., Davis, S. J., Liu, P., Pan, X., Williams, A. M., Iczkowski, K. A., ... Liang, M. (2017). Tissue-Specific MicroRNA Expression Patterns in Four Types of Kidney Disease. Journal of the American Society of Nephrology, 28(10), 2985-2992.

22.Zhang,J.,Yang,CL.,Zhang,L.,Zhou,B.Transcriptome analysis of podocyte to epithelial mesenchymal cell transformation in mouse high glucose memory model [J]. Chinese PLA medical journal, 2020,45 (02): 162-170

23. Zheng J, Wang W, Yu F, Dong P, Chen B, Zhou M-T.MicroRNA-30a suppresses the activation of hepaticstellate cells by inhibiting epithelial-to-mesenchymal transition.Cell Physiol Biochem. 2018;46(1):82-92.

24. Zhang lu,Wang ye,Li wenfeng,. MicroRNA-30a Regulation of Epithelial-Mesenchymal Transition in Diabetic Cataracts Through Targeting SNAI1[J]. Scientific reports, 2017, 7(1): 1117.

25. Yang, R., Hong, H., Wang, M., \& Ma, Z. (2018). Correlation Between Single-Nucleotide Polymorphisms Within miR-30a and Related Target Genes and Risk or Prognosis of Nephrotic Syndrome. DNA and cell biology, 37(3), 233-243.

26.Xie, K., Wang, C., Qin, N., Yang, J., Zhu, M., Dai, J., . . . Hu, Z. (2016). Genetic variants in regulatory regions of microRNAs are associated with lung cancer risk. Oncotarget, 7(30), 4796647974.

27.Sadeghi, H., Nazemalhosseini-Mojarad, E., Yaghoob-Taleghani, M., Amin-Beidokhti, M., Yassaee, V. R., Aghdaei, H. A., . . Mirfakhraie, R. (2018). miR-30a promoter variation contributes to the increased risk of colorectal cancer in an Iranian population. J Cell Biochem.

28.Wang, W., Zhang, H., Duan, X., Feng, X., Wang, T., Wang, P., . . Yang, Y. (2019). Association of genetic polymorphisms of miR-145 gene with telomere length in omethoate-exposed workers. Ecotoxicol Environ Saf, 172, 82-88. doi:10.1016/j.ecoenv.2019.01.023 
29. Wang, Y., Zheng, L., Wang, Z., Song, R., Yang, L., Geng, Y., . . Fang, W. (2018). Associations of gene- wide SNPs in SNAI1 and TWIST1 with breast cancer and ovarian cancer susceptibility among Chinese Han women. Oncology reports, 40(6), 3573-3584.

30. Yang, L., Wang, Y., Zheng, L., Jia, Y., Chen, Y., Chen, L., . . . Fang, W. (2016). Genetic Polymorphisms of TGFB1, TGFBR1, SNAI1 and TWIST1 Are Associated with Endometrial Cancer Susceptibility in Chinese Han Women. PloS one, 11(5), e0155270.

31.International Diabetes Federation. IDF Diabetes Atlas, 7 ed. Brussels, Belgium, 2015.

32.fkarian M, Sachs MC, Kestenbaum B, Hirsch IB, Tuttle KR, Himmelfarb J,et al. Kidney disease and increased mortality risk in type 2 diabetes.J Am SocNephrol.(2013) 24:302-8.

33.perkovic V, Jardine MJ, Neal B, Bompoint S, Heerspink HJL, Charytan DM, et al. Canagliflozin and Renal Outcomes in Type 2 Diabetes and Nephropathy. N Engl J Med 2019 Jun

13;380(24):2295-306

34.Lin YC,Chang YH, Yang SY, Wu KD, Chu TS. Update of pathophysiology and management of diabetic kidney disease. J Formos Med Assoc 2018 Aug;117(8):662-75.

35. Guan M, Keaton JM, Dimitrov L, Hicks PJ, Xu J, Palmer ND, et al. Genome-wide association study identifies novel loci for type 2 diabetes-attributed end-stage kidney disease in African Americans. Hum Genomics 2019 May 15;13(1):21.

36.Prunotto, M., Chaykovska, L., Bongiovanni, M., Frattini, M., Cagarelli, T., Weibel, F., . . Moll, S. (2017). Tubular Cytoplasmic Expression of Zinc Finger Protein SNAI1 in Renal Transplant Biopsies:

A Sign of Diseased Epithelial Phenotype? The American journal of pathology, 187(1), 55-69.

37.Tuttle, K., Bakris, G., Bilous, R., Chiang, J., de Boer, I., Goldstein-Fuchs, J., . . Molitch, M. (2014). Diabetic kidney disease: a report from an ADA Consensus Conference. American journal of kidney diseases : the official journal of the National Kidney Foundation, 64(4), 510-533. 38.Duan, X., Zhang, D., Wang, S., Feng, X., Wang, T., Wang, P., . . Yang, Y. (2020). Effects of polycyclic aromatic hydrocarbon exposure and miRNA variations on peripheral blood leukocyte DNA telomere length: A cross-sectional study in Henan Province, China. The Science of the total environment, 703,135600 .

39.Kalluri R, Neilson EG. Epithelial-mesenchymal transitionand its implications for fibrosis.J Clin Invest. 2003;112(12):1776-1784.22. Thiery JP, Acloque H, Huang RY, et al.

40. Epithelial-mesenchymal transitions in development and disease.Cell. 2009;139(5):871-890.23.

Das V, Bhattacharya S, Chikkaputtaiah C, et al.

41.The basics of epithelial-mesenchymal transition (EMT): a study from a structure, dynamics, and functional perspective.J Cell Physiol. 2019;234(9):14535-14555.

42. 55.Liu, Y.H. New insights into epithelial-mesenchymal transition in kidney fibrosis. JASN 2010, 21, 212-222.

43. Zheng J, Wang W, Yu F, Dong P, Chen B, Zhou M-T.MicroRNA-30a suppresses the activation of hepaticstellate cells by inhibiting epithelial-to-mesenchymal tran-sition.Cell Physiol Biochem.

2018;46(1):82-92.

44.Liu, Y.H. New insights into epithelial-mesenchymal transition in kidney fibrosis. JASN 2010, 21, 212-222. 
45. Barrallo-Gimeno, A., and Nieto, M.A. (2005). The Snail genes as inducers of cell movement and survival: implications in development and cancer. Development 132, 3151-3161.

46.Prunotto, M., Chaykovska, L., Bongiovanni, M., Frattini, M., Cagarelli, T., Weibel, F., ... Moll, S. (2017). Tubular Cytoplasmic Expression of Zinc Finger Protein SNAI1 in Renal Transplant Biopsies: A Sign of Diseased Epithelial Phenotype? The American journal of pathology, 187(1), 55-69.

Table 1 :General characteristics of patients with DKD and T2DM group

\begin{tabular}{|c|c|c|c|c|}
\hline & Variables & $\operatorname{T2DM}(n=280)$ & $\operatorname{DKD}(n=240)$ & $p$-value \\
\hline \multirow{10}{*}{$\begin{array}{l}\text { Demographic } \\
\text { characteristics }\end{array}$} & Age (years) & $60.01 \pm 9.83$ & $67.79 \pm 10.01$ & 0.000 \\
\hline & Gender(male/female) & $163 / 117$ & $120 / 120$ & 0.061 \\
\hline & Duriation of diabetes (years) & $9.67 \pm 5.67$ & $12.40 \pm 6.76$ & 0.000 \\
\hline & Duriation of hypertension (years) & $0(0-8.00)$ & $5.00(0-10.00)$ & 0.000 \\
\hline & BMI & $25.28 \pm 3.30$ & $24.88 \pm 3.94$ & 0.204 \\
\hline & SBP (mmHg) & $129.62 \pm 16.07$ & $138.24 \pm 18.49$ & 0.000 \\
\hline & $\mathrm{DBP}(\mathrm{mmHg})$ & $76.38 \pm 9.43$ & $76.50 \pm 10.96$ & 0.891 \\
\hline & $\mathrm{PP}(\mathrm{mmHg})$ & $53.05 \pm 12.21$ & $61.74 \pm 14.01$ & 0.000 \\
\hline & Smoking(yes/no) & $97 / 183$ & $54 / 186$ & 0.002 \\
\hline & Alcohol(yes/no) & $82 / 198$ & $45 / 195$ & 0.005 \\
\hline \multirow[t]{10}{*}{$\begin{array}{l}\text { Biochemical } \\
\text { indexes }\end{array}$} & FPG (mmol/l) & $8.956 \pm 3.40$ & $9.65 \pm 4.70$ & 0.052 \\
\hline & $\mathrm{HbA} 1_{C}(\%)$ & $8.37 \pm 2.05$ & $8.50 \pm 2.11$ & 0.492 \\
\hline & TC (mmol/l) & $4.54 \pm 1.39$ & $4.64 \pm 1.45$ & 0.388 \\
\hline & $\mathrm{TG}(\mathrm{mmol} / \mathrm{l})$ & $2.32 \pm 2.90$ & $2.16 \pm 2.27$ & 0.513 \\
\hline & HDL-C (mmol/l) & $1.15 \pm 0.33$ & $1.16 \pm 0.33$ & 0.915 \\
\hline & LDL-C (mmol/I) & $2.66 \pm 0.92$ & $2.76 \pm 1.07$ & 0.272 \\
\hline & $\mathrm{FIB}(\mathrm{g} / \mathrm{L})$ & $2.90 \pm 0.64$ & $3.25 \pm 0.73$ & 0.000 \\
\hline & $\mathrm{CRP}(\mathrm{mg} / \mathrm{l})$ & $1.39 \pm 1.85$ & $2.15 \pm 2.83$ & 0.000 \\
\hline & $\mathrm{ACR}(\mathrm{mg} / \mathrm{g} . \mathrm{cr})$ & $5.75(2.80-12.45)$ & $62.60(15.15-299.15)$ & 0.000 \\
\hline & eGFR(mL/min $\left./ 1.73 \mathrm{~m}^{2}\right)$ & $95.23 \pm 26.21$ & $60.16 \pm 26.96$ & 0.000 \\
\hline \multirow[t]{3}{*}{ drugs } & ACEI/ARB (yes/no) & $82 / 198$ & $126 / 114$ & 0.000 \\
\hline & Lipid lowering(yes/no) & $30 / 250$ & $43 / 197$ & 0.018 \\
\hline & Antiplatelet (yes/no) & $29 / 251$ & $59 / 181$ & 0.000 \\
\hline
\end{tabular}


T2DM: type 2 diabetes mellitus ; DKD : diabetic kidney disease; BMI: body mass index; alcohol: drinking; SBP: systolic blood pressure; DBP: diastolic blood pressure; FPG: fasting blood glucose; HbA1c: glycosylated hemoglobin; TC: total cholesterol; TG: triglycerides; HDL-C: high-density lipoprotein cholesterol;LDL-C:low-density lipoprotein cholesterol;CRP:C-reactive protein;FIB: Fibrinogen;ACR: urinary albumin / creatinine ratio; eGFR: estimated glomerular filtration rate; ACEI or ARB Use: angiotensin converting enzyme inhibitors or angiotensin II receptor blockers, $p$ value $<0.05$ indicates statistical signifificance.

Table2: mir-30a,snail SNP genotypes and allele frequency in DKD patients and control subjects

\begin{tabular}{|c|c|c|c|c|}
\hline Gene & Genotype & $\operatorname{DM}(n=280)$ & $\operatorname{DKD}(n=240)$ & p-Value \\
\hline \multirow{6}{*}{$\begin{array}{l}\text { miR-30a } \\
\text { Rs2222722 }\end{array}$} & TT & $36(129 \%)$ & $23(96 \%)$ & \\
\hline & CT & $148(52.9 \%)$ & $104(43.3 \%)$ & \\
\hline & $\mathrm{CC}$ & $96(34.3 \%)$ & $113(47.1 \%)$ & 0.012 \\
\hline & T allele & $220(39.3 \%)$ & 150(31.3\%) & \\
\hline & & & & 0.007 \\
\hline & C allele & $340(60.7 \%)$ & 330(68.7\%) & \\
\hline SNAI1 & $A A$ & $17(6.1 \%)$ & $14(5.8 \%)$ & \\
\hline \multirow[t]{5}{*}{ Rs1543442 } & $A G$ & $129(46.1 \%)$ & $88(36.7 \%)$ & \\
\hline & GG & $134(47.9 \%)$ & $138(57.5 \%)$ & 0.080 \\
\hline & A allele & $163(29.1 \%)$ & $116(24.2 \%)$ & \\
\hline & & & & 0.073 \\
\hline & G allele & $397(70.9 \%)$ & $364(75.8 \%)$ & \\
\hline
\end{tabular}

miR-30a:MicroRNA-30a; SNAI1:Snail family zinc finger 1; OR: odds ratio; $95 \% \mathrm{Cl}$ : 95\% confidence interval,p value $<0.05$ indicates statistical signifificance.

Table3: Associations of miR-30a and SNAI1 genotypes with DKD in diabetic patients.

\begin{tabular}{|c|c|c|c|c|c|}
\hline \multirow[t]{2}{*}{ Gene } & \multirow[t]{2}{*}{ Genotype } & \multicolumn{2}{|c|}{ Crude model } & \multicolumn{2}{|c|}{ Adjusted model $^{\mathrm{a}}$} \\
\hline & & OR $(95 \% \mathrm{Cl})$ & $p$-Value & OR $(95 \% \mathrm{Cl})$ & $\begin{array}{l}\mathrm{p}- \\
\text { Value }\end{array}$ \\
\hline miR-30a & TT & reference & 0.012 & reference & 0.001 \\
\hline \multicolumn{6}{|c|}{ Rs2222722 } \\
\hline & CT & $1.10(0.62-1.97)$ & 0.748 & $1.79(0.63-5.10)$ & 0.276 \\
\hline & CC & $1.84(1.02-3.23)$ & 0.042 & $4.56(1.58-13.15)$ & 0.005 \\
\hline & Dominant(TT vs. CT+CC) & $1.39(0.80-2.42)$ & 0.242 & $2.80(1.04-7.56)$ & 0.042 \\
\hline & Recessive(CT+TT vs. CC) & $1.70(1.20-2.43)$ & 0.003 & $2.81(1.58-4.97)$ & $<0.001$ \\
\hline & Allele (T vs C) & $1.42(1.10-1.84)$ & 0.007 & $2.11(1.40-3.19)$ & $<0.001$ \\
\hline
\end{tabular}


SNAI1

AA

reference

0.081

reference

0.084

Rs1543442

$A G$

$0.83(0.39-1.77)$

0.626

$0.51(0.14-1.88)$

0.310

GG

1.25(0.59-2.64)

0.557

$0.94(0.26-3.40)$

0.930

Dominant(AAvs.AG+GG)

1.04(0.50-2.16)

0.909

$0.75(0.21-2.65)$

0.650

Recessive(AA+AGvs.GG)

1.47(1.04-2.09)

0.028

1.73(1.01-2.96)

0.046

Allele (A vs $G$ )

1.29(0.98-1.70)

0.073

1.37(0.88-2.13)

0.159

Abbreviations:OR: odd ratio; 95\% Cl: 95\% confidence interval.Data were calculated by logistic regression analysis with adjusted for age, gender, duration of diabetes, duration of hypertension, BMI, fasting blood glucose, glycosylated hemoglobin, TC, TG, HDL-C, LDL-C, systolic blood pressure, diastolic blood pressure, mean arterial pressure, smoking, drinking, ACEI / ARB drugs,p value $<0.05$ indicates statistical signifificance.

Table 4: Stratified analysis of the association between the mir-30a SNAIL SNP and risk of DKD

\begin{tabular}{|c|c|c|c|c|c|}
\hline \multirow{2}{*}{ Gene } & & \multirow{2}{*}{ Gender } & \multirow{2}{*}{ Genotype } & \multicolumn{2}{|c|}{ T2DM-group vs. DKD-group } \\
\hline & & & & $\mathrm{OR}(95 \% \mathrm{Cl})$ & $\mathrm{p}$-Value \\
\hline \multirow{10}{*}{$\begin{array}{l}\text { miR-30a } \\
\text { Rs2222722 }\end{array}$} & Age (years) & $<65$ & $\mathrm{CT}+\mathrm{TT}$ vs. $\mathrm{CC}$ & $2.52(1.48-4.28)$ & 0.001 \\
\hline & & $\geq 65$ & $\mathrm{CT}+\mathrm{TT}$ vs. $\mathrm{CC}$ & $1.590(0.93-2.73)$ & 0.092 \\
\hline & gender & Male & $\mathrm{CT}+\mathrm{TT}$ vs. $\mathrm{CC}$ & $1.85(1.15-2.98)$ & 0.012 \\
\hline & & Female & $\mathrm{CT}+\mathrm{TT}$ vs. $\mathrm{CC}$ & $1.67(0.98-2.86)$ & 0.060 \\
\hline & $\begin{array}{l}\text { Duration } \\
\text { ofdiabetes }\end{array}$ & $<10$ & $\mathrm{CT}+\mathrm{TT}$ vs. $\mathrm{CC}$ & $1.75(1.01-3.07)$ & 0.048 \\
\hline & & $\geq 10$ & $\mathrm{CT}+\mathrm{TT}$ vs. $\mathrm{CC}$ & $1.67(1.04-2.67)$ & 0.032 \\
\hline & $\mathrm{HbA} 1_{c}(\%)$ & $<8$ & $\mathrm{CT}+\mathrm{TT}$ vs. $\mathrm{CC}$ & $1.61(0.98-2.66)$ & 0.060 \\
\hline & & $\geq 8$ & $\mathrm{CT}+\mathrm{TT}$ vs. $\mathrm{CC}$ & $1.82(1.10-3.02)$ & 0.020 \\
\hline & $\mathrm{BMI}(\mathrm{kg} / \mathrm{m} 2)$ & $<24$ & $\mathrm{CT}+\mathrm{TT}$ vs. $\mathrm{CC}$ & $1.20(1.11-3.60)$ & 0.021 \\
\hline & & $\geq 24$ & $\mathrm{CT}+\mathrm{TT}$ vs. $\mathrm{CC}$ & $1.53(0.97-2.39)$ & 0.065 \\
\hline \multirow{4}{*}{$\begin{array}{l}\text { SNAI1 } \\
\text { Rs1543442 }\end{array}$} & Age (years) & $<65$ & $A A+A G$ vsGG & $1.47(0.88-2.48)$ & 0.145 \\
\hline & & $\geq 65$ & $A A+A G$ vs $G G$ & $1.23(0.74-2.05)$ & 0.429 \\
\hline & gender & male & $A A+A G$ vsGG & $1.64(1.01-2.64)$ & 0.044 \\
\hline & & Female & $A A+A G$ vsGG & $1.33(0.80-2.22)$ & 0.272 \\
\hline
\end{tabular}




\begin{tabular}{|c|c|c|c|c|}
\hline \multicolumn{5}{|l|}{$\begin{array}{l}\text { Duration } \\
\text { ofdiabetes }\end{array}$} \\
\hline & $\geq 10$ & $A A+A G$ vsGG & $1.26(0.80-1.99)$ & 0.316 \\
\hline \multirow[t]{2}{*}{$\mathrm{HbA} 1_{C}(\%)$} & $<8$ & $A A+A G$ vsGG & $1.91(1.16-3.15)$ & 0.011 \\
\hline & $\geq 8$ & $A A+A G$ vsGG & $1.17(0.72-1.91)$ & 0.532 \\
\hline \multirow[t]{2}{*}{$\mathrm{BMI}(\mathrm{kg} / \mathrm{m} 2)$} & $<24$ & $A A+A G$ vsGG & $1.78(1.01-3.15)$ & 0.048 \\
\hline & $\geq 24$ & $A A+A G$ vsGG & $1.28(0.82-2.00)$ & 0.269 \\
\hline
\end{tabular}

Abbreviations:OR: odd ratio;95\% Cl: 95\% confidence interval. HbA1C, haemoglobin A1C; BMI:body mass index,p value $<0.05$ indicates statistical signifificance.

Table 5: Association between SNP genotypes and the risk of $A C R \geq 30(\mathrm{mg} / \mathrm{g} . \mathrm{cr})$ and eGFR $\leq 60$ $(\mathrm{mL} / \mathrm{min} / 1.73 \mathrm{~m} 2)$ based on unconditional logistic regression model analysis.

\begin{tabular}{|c|c|c|c|c|c|}
\hline \multirow[t]{2}{*}{ Gene } & \multirow[t]{2}{*}{ Genetic models } & \multicolumn{2}{|c|}{$\begin{array}{l}\text { ACR }<30(\mathrm{mg} / \mathrm{g} . \mathrm{cr}) \text { vs } \\
A C R \geq 30(\mathrm{mg} / \mathrm{g} . \mathrm{cr})\end{array}$} & \multicolumn{2}{|c|}{$\begin{array}{l}\text { eGFR>60 }(\mathrm{mL} / \mathrm{min} / 1.73 \mathrm{~m} 2) \\
\text { vs } \\
\text { eGFR } \leq 60(\mathrm{~mL} / \mathrm{min} / 1.73 \mathrm{~m} 2)\end{array}$} \\
\hline & & OR (95 \% Cl) & p-Value & OR (95 \% Cl) & $\mathrm{p}$-Value \\
\hline miR-30a & Dominant(TT vs. $\mathrm{CT}+\mathrm{CC})$ & $1.02(0.99-1.03)$ & 0.107 & $1.39(0.70-2.74)$ & 0.347 \\
\hline \multirow[t]{2}{*}{ Rs2222722 } & Recessive(CT+TT vs. CC) & $1.59(1.08-2.34)$ & 0.019 & $2.13(1.37-3.32)$ & 0.001 \\
\hline & Allele (T vs C) & $1.43(1.07-1.91)$ & 0.015 & $1.59(1.15-2.18)$ & 0.005 \\
\hline \multirow{3}{*}{$\begin{array}{l}\text { SNAI1 } \\
\text { Rs1543442 }\end{array}$} & Dominant (AAvs $A G+G G)$ & $114(049-261)$ & 0764 & $104(0$ 39-2 79) & 0946 \\
\hline & Recessive(AA+AG vs.GG) & $1.19(0.81-1.74)$ & 0.383 & $1.44(0.99-2.10)$ & 0.080 \\
\hline & Allele (A vs G) & $1.14(0.83-1.55)$ & 0.419 & $1.30(0.91-1.84)$ & 0.149 \\
\hline
\end{tabular}

Abbreviations:OR: odd ratio;95\% Cl: 95\% confidence interval,adjusted for age and gender;ACR: urinary albumin / creatinine ratio; eGFR: estimated glomerular filtration rate. Table 6: Results of DKD multifactor and non-conditional logistic regression analysis

\begin{tabular}{llllll}
\hline Independent variables & $\beta$ & $\mathrm{SE}$ & $\mathrm{P}$ value & $\mathrm{OR}$ & $95 \% \mathrm{Cl}$ \\
\hline Age(year) & 0.076 & 0.019 & 0.000 & 1.079 & $1.040-$ \\
& & & & & $\begin{array}{l}1.120 \\
0.206-\end{array}$ \\
Gender(male/female) & -0.806 & 0.395 & 0.041 & 0.447 & 0.968 \\
& & & & & $1.275-$ \\
FIB $(\mathrm{g} / \mathrm{L})$ & 0.689 & 0.228 & 0.002 & 1.992 & 3.112
\end{tabular}




\begin{tabular}{lccccc}
\hline $\mathrm{CC}(\mathrm{rs} 22222)$ & 1.247 & 0.311 & 0.000 & 3.480 & $\begin{array}{l}1.892- \\
6.398\end{array}$ \\
$\mathrm{GG}(\mathrm{rs} 1543442)$ & 0.801 & 0.296 & 0.007 & 2.228 & $\begin{array}{l}1.247- \\
3.980 \\
1.314-\end{array}$ \\
ACEI/ARB use (yes/no) & 0.913 & 0.327 & 0.005 & 2.493 & 4.729 \\
\hline
\end{tabular}

Abbreviations:OR: odd ratio; 95\% Cl: 95\% confidence interval;CC(rs2222722):miR-30a rs2222722 CC genotype; GG(rs1543442):SNAILrs1543442GG genotype;ACEI or ARB Use: angiotensin converting enzyme inhibitors or angiotensin II receptor blockers, $p$ value $<0.05$ indicates statistical signifificance.

Table 7: Combined effect of mir-30a rs2222722 and SNAIL rs1543442 Polymorphisms on DKD.

\begin{tabular}{|c|c|c|c|}
\hline Genotypes & & DM-group vs.DKD-group & \\
\hline miR-30a & SNAIL & OR $(95 \% \mathrm{Cl})$ & $P$ \\
\hline \multirow[t]{3}{*}{$\mathrm{CC}$} & $A A$ & reference & \\
\hline & AG & $0.51(0.09-2.89)$ & 0.445 \\
\hline & GG & $0.69(0.12-4.00)$ & 0.683 \\
\hline \multirow[t]{3}{*}{$\mathrm{CT}+\mathrm{TT}$} & GG & reference & \\
\hline & AG & $0.46(0.27-0.76)$ & 0.003 \\
\hline & $A A$ & $0.74(0.32-1.74)$ & 0.492 \\
\hline
\end{tabular}

Abbreviations:OR: odds ratio; $95 \% \mathrm{Cl}$ : 95\% confidence interval, $\mathrm{p}$ value $<0.05$ indicates statistical signifificance. 



\section{Supplementary Files}

This is a list of supplementary files associated with this preprint. Click to download.

- supplementaryinformation.docx 